\title{
Stem cells in prostate cancer initiation and progression
}

\author{
Devon A. Lawson ${ }^{1}$ and Owen N. Witte ${ }^{1,2,3}$
}

1Department of Microbiology, Immunology and Molecular Genetics, and 'Department of Molecular and Medical Pharmacology, David Geffen School of Medicine, and ${ }^{3}$ Howard Hughes Medical Institute, UCLA, Los Angeles, California, USA.

\begin{abstract}
Peter Nowell and David Hungerford's discovery of the Philadelphia chromosome facilitated many critical studies that have led to a paradigm shift in our understanding of cancer as a disease of stem cells. This Review focuses on the application of these concepts to investigation of the role of stem cells in prostate cancer initiation and progression. Major strides in the development of in vitro and in vivo assays have enabled identification and characterization of prostate stem cells as well as functional evaluation of the tumorigenic effects of prostate cancer-related genetic alterations.
\end{abstract}

Discovery and study of the Philadelphia chromosome have provided the foundation for many of our fundamental concepts about cancer today (1). The Philadelphia chromosome is a reciprocal translocation between the long arms of chromosomes 9 and 22 $[\mathrm{t}(9 ; 22)(\mathrm{q} 34 ; \mathrm{q} 11)]$ that is present in most human chronic myelogenous leukemia (CML) patients (2). This translocation causes fusion of the breakpoint cluster region $(B C R)$ with portions of the $\mathrm{v}$-Abl Abelson murine leukemia viral oncogene homolog $(A B L)$ gene (reviewed in ref. 3). The resulting chimeric oncoprotein, BCR$\mathrm{ABL}$, possesses aberrant tyrosine kinase activity (3). These findings have demonstrated the role of genetic change, genomic instability, and tyrosine kinase signaling in tumorigenesis and have highlighted BCR-ABL as an important therapeutic target.

Discovery of the Philadelphia chromosome also facilitated several key studies that have led to a paradigm shift in our understanding of cancer as a disease of stem cells. During chronic phase CML, the Philadelphia chromosome is found in many different blood cell lineages $(4,5)$. This implies that it must arise either in a stem or progenitor cell capable of giving rise to each lineage or independently in cells of each lineage. Philip Fialkow and colleagues addressed this issue in a clever series of experiments examining Philadelphia chromosome-positive $\left(\mathrm{Ph}^{+}\right)$leukemic cells for the expression of an X chromosome-linked gene, glucose-6-phosphate dehydrogenase $(4,5)$. Each cell in the body only expresses one allele for this gene as a result of the inactivation of one $\mathrm{X}$ chromosome during development. Interestingly, all $\mathrm{Ph}^{+}$cells in female patients heterozygous for the gene expressed the same allele $(4,5)$. Although it is possible that $\mathrm{X}$ chromosome inactivation status may change in cancer cells during tumor progression, this finding strongly suggests human CML is clonal in origin and therefore must originate in a stem cell. Subsequent studies using surface antigens to purify HSCs have confirmed that the Philadelphia chromosome is present in HSCs $(6,7)$.

More recent studies have demonstrated that stem cells also sustain disease in later phases of malignancy. Observations that only

Nonstandard abbreviations used: $\mathrm{ABL}, \mathrm{v}-\mathrm{Abl}$ Abelson murine leukemia viral oncogene homolog; $\mathrm{AR}$, androgen receptor; $\mathrm{BCR}$, breakpoint cluster region; CK8, cytokeratin 8; CML, chronic myelogenous leukemia; CSC, cancer stem cell; HRPC, hormone-refractory prostate cancer; LSC, $\mathrm{Lin}(\mathrm{CD} 45 / \mathrm{CD} 31 / \mathrm{Ter} 119)^{-} \mathrm{Sca}-1^{+} \mathrm{CD} 49 \mathrm{f}^{+}$; $\mathrm{Ph}^{+}$, Philadelphia chromosome-positive; PIN, prostatic intraepithelial neoplasia; PSC, prostate stem cell; Sca-1, stem cell antigen-1; TF, transcription factor.

Conflict of interest: The authors have declared that no conflict of interest exists. Citation for this article: J. Clin. Invest. 117:2044-2050 (2007). doi:10.1172/JCI32810. a minor subpopulation of cells in tumor samples have the capacity to initiate clonal growth in in vitro cultures or in in vivo transplant models had perplexed researchers for over 50 years (reviewed in refs. 8,9$)$. Two theories were proposed to explain this paradox. The stochastic theory suggested that all cancer cells are equally malignant but only clones that randomly possess favorable biological properties will grow upon transplantation. An alternative theory predicted that tumors are hierarchical like normal tissues and only the rare subpopulation of cells at the pinnacle of that hierarchy have the unique biological properties necessary for tumor initiation $(8,9)$. Studies by John Dick and colleagues provided evidence for the hierarchy model. This group demonstrated that only the small subpopulation $(0.1 \%-1.0 \%)$ of $\mathrm{Lin}^{-} \mathrm{CD} 34^{+} \mathrm{CD} 38^{-}$cells within human acute myelogenous leukemia samples were capable of initiating disease when transplanted into immune-deficient mice (10). These cells possessed the same antigenic profile as normal human HSCs, which are at the pinnacle of the normal hematopoietic hierarchy. This population also had the unique capacity to selfrenew to propagate the disease as well as differentiate to produce the many leukemic cell types represented in the original leukemia. Since these cancer cells possess properties unique to normal tissue stem cells, they have been termed "cancer stem cells" (CSCs).

These studies make it clear that stem cells play critical roles in both the initiation and maintenance of human leukemia. But does this mean stem cells are exclusively competent to perform these functions? Huntly et al. (11) showed that BCR-ABL can only initiate CML in mice when expressed in HSCs and not in committed progenitor cells. Inactivation of the AP-1 transcription factor (TF) JunB, a transcriptional regulator of myelopoiesis and potential tumor suppressor, is also only sufficient to induce disease in HSCs (12). However, leukemia-associated fusion oncogenes such as MOZ-TIF2, MLL-ENL, and MLL-AF9 will induce disease from both HSCs as well as myeloid progenitors $(11,13,14)$. To determine why MOZ-TIF2 but not BCR-ABL can transform committed progenitors, Huntly et al. examined the in vitro self-renewal activity of progenitors expressing each oncogene and found that $M O Z-T I F 2$ is sufficient to restore self-renewal in committed progenitors while $B C R-A B L$ is not (11). MLL-AF9 similarly can confer self-renewal activity in committed progenitors. Microarray studies comparing the expression profile of leukemic granulocyte-macrophage progenitors (GMPs) expressing MLL-AF9 with normal HSCs and progenitor cells showed that leukemic GMPs maintain an expression profile similar to that of normal GMPs but reacquire 


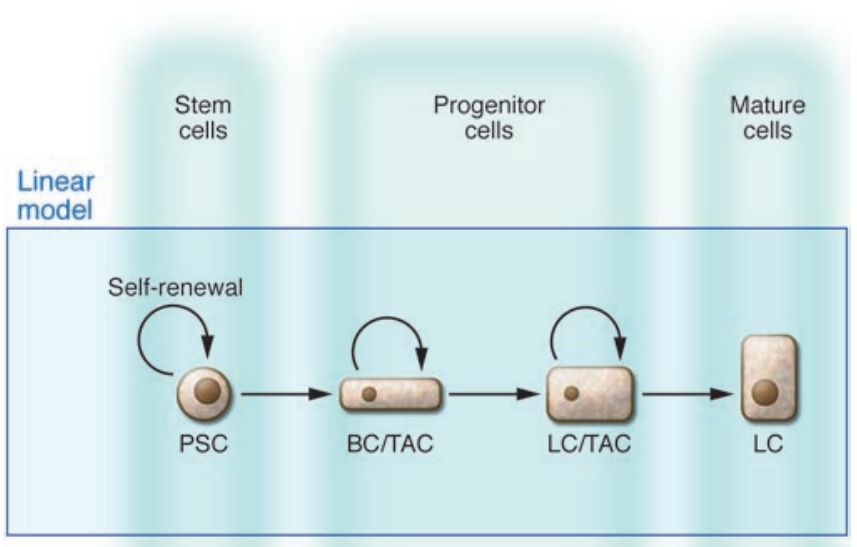

Bifurcated

model

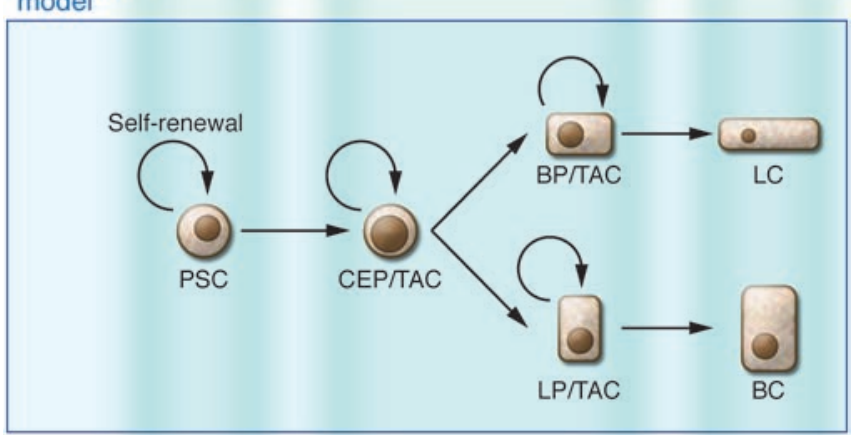

\section{Figure 1}

Models of prostate epithelial cell differentiation. In traditional linear hierarchy models, self-renewing PSCs residing in the basal cell (BC) layer give rise to transit-amplifying cells (TACs) of intermediate phenotypes that may express both basal and luminal cell markers during their maturation. These cells theoretically possess transient self-renewal activity and produce large numbers of terminally differentiated secretory luminal cells (LCs). In bifurcated models, basal cells and luminal cells represent separate epithelial cell lineages. These lineages may be sustained by intermediate transit-amplifying cells and/or lineage-restricted basal and luminal cell progenitors (BP and LP, respectively).

a self-renewal-associated transcription program characteristic of normal HSCs (13). These studies demonstrate it is the self-renewal activity of stem cells that makes them more conducive to oncogenesis. Acquisition of this property by other cells, however, appears to enable them to support tumor initiation.

The role of stem cells is now being addressed in many solid tissue cancers. Although prostate cancer is now the most commonly diagnosed cancer in American men, the etiology of this disease remains unclear (15). Interestingly, several aspects of this disease are remarkably similar to CML. CML is most frequently diagnosed in adults and the elderly and first presents as a chronic myelogenous hyperplasia that eventually progresses to acute immature blast crisis following the accumulation of genetic and epigenetic alterations in addition to $B C R-A B L$. Prostate cancer is similarly a disease of aging that is thought to begin with the chronic accumulation of prostatic intraepithelial neoplasia (PIN) lesions that may eventually develop into adenocarcinoma, although no experimental evidence for this has yet been provided. Many adenocarcinomas also progress to a poorly differentiated, hormone-refractory prostate cancer (HRPC) consisting mostly of immature blast-like cells. Arul Chinnaiyan and colleagues have recently identified a class of translocations that, like $B C R-A B L$, are present in the majority of prostate cancers (16). These translocations result in fusion of the androgen-regulated promoter for TMPRSS2 with the coding regions of ETS family TFs that are associated with Ewing sarcoma and several types of human leukemia $(17,18)$. In light of these findings, a major goal of our laboratory is application of the fundamental concepts learned about stem cells in CML to elucidate prostate cancer etiology. This Review will focus on current concepts of stem cells in prostate cancer initiation and progression as well as the implication of these concepts in prostate cancer research and therapeutic development. We hope that understanding the cellular basis of prostate cancer will yield insight critical for the development of more efficacious therapeutics to fight the disease.

\section{The debate: existing evidence for the cell of origin of prostate cancer}

Anatomy of the prostate gland. The prostate is a glandular organ comprised of three anatomically distinct epithelial cell populations that may contribute to tumorigenesis (reviewed in ref. 19). Prostate basal cells form a layer along the basement membrane of each prostatic duct. Neuroendocrine cells also reside along the basement membrane and secrete neuroendocrine peptides that support epithelial growth and viability. Luminal cells form a layer just above the basal cells and secrete prostatic proteins into the lumenal space. The prostate also contains several types of stromal cells including fibroblasts, myofibroblasts, and smooth muscle cells that guide the growth and differentiation of the epithelium. Blood cell elements as well as vascular and stromal endothelial cells are also present in the gland.

Classic androgen cycling experiments by John Isaacs and colleagues suggest the prostate epithelium must contain a stem cell population. When rodents are deprived of androgen by surgical or medicinal castration, the gland atrophies due to the apoptosis of terminally differentiated cells dependent on androgen for viability (20). When androgen is replaced, the gland regenerates and resumes normal secretory function. Isaacs's group showed that this involution and regeneration could be repeated for many sequential cycles (21). This showed that a stem cell population with profound capacity for self-renewal and differentiation must exist within the gland.

Localization of stem cells in the prostate. The preferential survival of basal cells following androgen ablation has led to the traditionally held hypothesis that prostate stem cells (PSCs) reside within the basal cell layer of the gland (20). This is supported by findings that mice null for the basal cell marker $p 63$ are born without the prostate or mammary gland (22-25). Chimeric animals created from $p 63^{-/-}$blastocysts complemented with $p 63^{+++} \beta$-galactosidase-positive ES cells also produce only $\beta$-galactosidase-positive luminal cells (26). Human basal cells also express BCL-2, an antiapoptotic protein that is commonly expressed by tissue stem cells (27). BrdU pulse chase experiments performed by Tsujimura et al. (28), however, show that long-term label-retaining cells localize to a specific region of the gland that is proximal to the urethra. This region is characterized by a thick band of morphologically distinct smooth muscle cells that secrete high levels of TGF- $\beta$, making it a compelling location for the PSC niche since this factor is known to 
promote stem cell quiescence (29). Interestingly, both basal and luminal cells in the proximal region retain the label after many cycles of androgen ablation and add-back, suggesting stem cells may not be restricted to the basal layer. Cells from this region possess enhanced growth activity both in vitro and in vivo, and serial transplant studies have shown that these cells can self-renew to regenerate prostate tissue de novo for at least 4 passages $(28,30)$.

Models of prostate epithelial differentiation. The traditional model for prostate epithelial differentiation proposes that PSCs residing in the basal cell layer give rise to intermediate, transit-amplifying cells that produce large numbers of terminally differentiated secretory luminal cells (31). This model implies a linear differentiation scheme in which basal and luminal cells comprise one lineage and basal cells are essentially luminal cell progenitors (Figure 1). This hypothesis is supported by the existence of cells of intermediate phenotype that express both basal- and luminal cell-specific cytokeratins in both fetal and adult stages of prostate development (32-34). Intermediate cells can also be identified in in vitro cultures of primary prostate epithelium (34-37). Several studies have also suggested basal cells can differentiate into luminal cells in vitro (38-40).

Alternative theories for prostate epithelial differentiation propose basal and luminal cells may represent separate epithelial lineages (Figure 1). This is similar to prevailing models for epithelial differentiation in the mammary gland, a tissue that is anatomically and functionally analogous to the prostate (reviewed in ref. 41). Recent studies by Gerald Cunha and colleagues showed that fetal urogenital sinus tissue from p63-null mice can regenerate prostate ductal tissue following implantation in immunodeficient mice (42). Regenerated prostate tissue lacked phenotypically identifiable basal cells but did contain cells that expressed typical luminal cell markers and had secretory function. This presented the possibility that luminal cells arise in the absence of basal cells. However, there are other explanations for these observations, one example being that loss of $\mathrm{p} 63$ expression causes differentiation defects that make basal cells unidentifiable in regenerated tissue.

BrdU-labeling experiments by Tsujimura et al. (28) have demonstrated that the dramatic growth of the murine gland that occurs during puberty is due to the rapid proliferation of a subset of luminal cells, although the majority of basal cells do not divide. This suggests that basal and luminal cell progenitors may also exist that can respond independently to growth cues. This would also explain why subsets of luminal cells survive in the absence of androgen after castration (20) and would correspond with recent demonstration by Asselin-Labat et al. (43) of a luminal-restricted progenitor population in the mammary gland. One limitation of each of these models is that they do not address the ontogeny of neuroendocrine cells in the prostate. Neuroendocrine cells may represent a subset of the basal cell lineage, a unique epithelial lineage, or even a lineage produced from a separate stem cell population (44).

The cell of origin of prostate cancer. Most speculation over the cellular origin of human prostate cancer comes from correlative studies examining the cellular composition of prostate tumors. In human prostate adenocarcinomas, the majority of cancer cells express luminal cell-specific markers such as cytokeratin 8 (CK8), CK18, and prostate-specific antigen (45). Cells that solely express basal cell markers such as CK5, CK14, and p63 are rarely observed. This has led some to suggest that prostate cancers are derived from a luminal cell progenitor or mature luminal cell that has acquired self-renewal activity through mutation. Some reports, however, have identified intermediate cells that coexpress both basal and luminal cell markers within prostate cancers (27). PSCA, a putative marker of normal late-intermediate prostate cells, is also often upregulated in prostate cancers $(39,46)$. These data indicate the disease may originate in an intermediate or transit-amplifying epithelial cell that precedes luminal cell differentiation.

Several properties of human prostate cancers suggest the disease may instead arise from a PSC. The progression to androgenindependent HRPC during androgen ablation therapy has led to speculation that prostate tumors may contain a small population of androgen-independent cells that survive and can expand in the absence of androgen (reviewed in ref. 47). Since normal PSCs are androgen independent, it is reasonable to suspect they may be the source of these cells. Recent efforts to identify and characterize prostate CSCs also support this hypothesis. Collins et al. (48) demonstrated that the primary human prostate cancer cell subpopulation with the highest in vitro proliferative potential is negative for androgen receptor (AR) expression, as is suspected for normal PSCs. This population also possesses a CD $44^{+} \alpha_{2} \beta_{1}{ }^{\text {hi }} \mathrm{CD} 133^{+}$antigenic profile characteristic of normal human PSCs and predominantly expresses the basal cell cytokeratins $(48,49)$. Using several human prostate xenograft tumors and cell lines, Patrawala et al. (50) have also demonstrated that the $\mathrm{CD} 44^{+}$population within these models displays enhanced proliferative activity in vitro and increased tumor-initiating and metastatic activity in vivo. These $\mathrm{CD} 44^{+}$cells are likewise $\mathrm{AR}^{-}$and express higher mRNA levels of several "stemness" genes, including OCT3/4, BMI1, $\beta$-CATENIN, and SMOOTHENED.

The lineage status of CSCs has also been addressed by several other groups using human telomerase reverse transcriptaseimmortalized primary human prostate cancer cell lines $(51,52)$. Gu et al. (51) showed that, as with CSCs, these clonally derived cell lines could regenerate prostate tumors in mice that resembled the original patient tumor with respect to histopathology and Gleason score. Regenerated tumors also contained basal, luminal, and neuroendocrine-like cancer cells, suggesting the clone of origin of the lines had multilineage differentiation capacity. Analysis of these lines showed they are predominantly $\mathrm{AR}^{-}$and $\mathrm{p}^{6} 3^{-}$and express the pluripotency markers Oct4, Nanog, and Sox2, as well as the progenitor markers CD44, CD133, and CD117 (also known as c-Kit).

An interesting question generated from these findings is whether the TMPRSS2-ETS fusion gene is expressed in CSCs if they are in fact $\mathrm{AR}^{-}$. Fusion of the androgen-regulated TMPRSS2 promoter with the coding region of various ETS TFs is thought to result in TF overexpression (16). ETS-regulated target genes have numerous potentially oncogenic functions, including the regulation of proliferation, differentiation, apoptosis, angiogenesis, and transformation. If PSCs and CSCs are $\mathrm{AR}^{-}$, it is likely they do not express the fusion transcript. Does this mean that PSCs are not the origin of human prostate cancer, or that the fusion gene is not an initiating event but instead a secondary event generated through genomic instability? Recent identification of the TMPRSS2-ETS fusion gene in approximately $20 \%$ of PIN lesions suggests that its formation may be an early event in tumorigenesis (53). Perhaps it serves as a "first hit" that takes place in a primitive $\mathrm{AR}^{-}$cell but does not take effect until it is expressed in differentiated progeny that are $\mathrm{AR}^{+}$. Several studies have shown that some HSCs may not express BCR-ABL even though they contain the fusion gene. Expression of the fusion protein in the progeny of such HSCs is, however, cancer promoting. Research to answer these critical questions will provide invaluable insight into the etiology of prostate cancer. 
Several laboratories have begun to investigate prostate cancer initiation using mouse models of the disease. As in human prostate cancer, disease in the Pten prostate-specific knockout mouse model progresses from PIN to invasive adenocarcinoma and metastasis (54). Although Cre mediates Pten deletion in both basal and luminal cells, disease progression is associated with a disproportionate expansion of basal cells, indicating disease in this model may initiate and be sustained by primitive cells within the basal cell population (55). Zhou et al. (56) also suggest that disease in their $\mathrm{Rb}^{-/-} \mathrm{p53^{-/- }}$ prostate-specific knockout model initiates from primitive cells. The earliest invasive neoplasms that develop in these mice express stem cell antigen-1 (Sca-1) and are observed in the proximal region of the gland, which is characteristic of murine prostate stem and progenitor cells. In contrast to the PTEN knockout model, the cells that comprise these lesions express luminal and neuroendocrine markers but are negative for basal cell markers like CK5. The authors of this study further found that both proximal and distal cells will regenerate tumors when engrafted under the kidney capsule of immunodeficient mice. This suggests that microenvironmental factors unique to the proximal region of the gland may promote cancer formation in this model.

Functional studies to directly compare the tumorigenic potential of different prostate cell types are necessary to resolve this debate over the cell of origin of prostate cancer. Several technical advancements are necessary for these studies: (a) development of reliable in vitro and in vivo assays for prostate epithelial cell growth; (b) identification of PSCs and epithelial lineage hierarchy; and (c) development of methods to model prostate cancer from dissociated populations of prostate cells. The remainder of this Review will focus on current efforts to achieve these goals.

\section{Development of prostate epithelial stem cell assays}

The in vivo dissociated prostate cell regeneration system. Most in vivo assays for the growth of normal adult rodent epithelial cells are based on the tissue recombination methods developed by Cunha and Lung (57). In this system, tissue fragments of fetal urogenital sinus mesenchyme are used to support the growth of normal prostate epithelial tissue fragments when implanted in collagen under the renal capsule of immunodeficient mice. In order to evaluate the growth activities of different prostate cell subpopulations, we have modified this system using mechanical and enzymatic digestion to dissociate both the urogenital sinus mesenchyme and adult murine prostate tissue into single cell suspensions (58). Dissociated prostate epithelia regenerate ductal structures that histologically resemble normal murine prostate. Prostate tissue can also be regenerated when dissociated cells are implanted s.c. in Matrigel or in collagen onto the flanks of immunodeficient mice $(59,60)$. Experiments utilizing mixed populations of differentially marked epithelial cells have demonstrated that the tubules regenerated using these protocols are clonal, as chimeric tubules expressing both markers are rarely observed (60-62). Immunohistochemical analyses have shown that each duct contains both basal and luminal cell lineages $(58,60-62)$. Since regenerated ducts are clonal, this suggests that tubule-regenerating cells possess the capacity for multilineage differentiation.

To investigate PSC self-renewal, Lynette Wilson and colleagues have utilized a serial transplantation approach analogous to the serial reconstitution method used to measure HSC self-renewal (30). This group demonstrated that regenerated prostate tissue can be dissociated and transplanted to regenerate prostate tissue at least three times, indicating regenerated ducts contain cells capable of self-renewing to support several rounds of prostate regeneration. Interestingly, the regenerative activity of transplanted cells drops almost 100 -fold from generation one to three. This may be because many cells within regenerated tissue represent short-term progenitors with limited regenerative activity that eventually expire during serial transplantation. Alternatively, the diminution in regenerative activity may be due to loss of regenerating cells during tissue digestion at each passage.

In vitro prostate colony assays. Epithelial cells from many organs such as the skin and mammary gland can be grown as colonies in two dimensional culture conditions $(35,63)$. Human prostate epithelial cells can also be cultivated in this manner when cocultured with irradiated fibroblast feeder layers in low-calcium, serum-free medium containing growth factors such as FGF and EGF, which are known to promote stem cell maintenance (31). Under these conditions, murine prostate epithelial cells form colonies of cells that express epithelial cytokeratins when cultured with irradiated 3T3 feeder cells (64). These colonies appear to be clonogenic, as differential marking experiments have shown that chimeric colonies rarely appear. Castration was also shown to enrich for colony-forming cells, suggesting that colonies arise predominantly from primitive prostate epithelia. These findings demonstrate that this assay can be used to quantitatively compare stem cell enrichment strategies.

In vitro prostate sphere assays. Although colonies appear to be derived from primitive cells, they do not passage efficiently since this culture condition appears to promote differentiation. Stem cells from the central nervous system and mammary gland have been maintained by culturing them in nonadherent conditions as three-dimensional spheres $(65,66)$. Human prostate cancer cells lines and immortalized lines of normal cells can form spheroid structures when embedded in a solubilized basement membrane preparation called Matrigel (67-69). We have developed a murine prostate sphere-forming assay using similar conditions (64). The majority of spheres generated in these conditions are composed of several epithelial cell layers that surround a hollow center. We speculate that this is analogous to the canalization process that occurs during prostate duct development.

Like the colony assay, differential marking and castration studies have shown that prostate spheres are of clonal origin and derive from primitive cells (64). Spheres can be dissociated and passaged for over 10 generations, suggesting they contain a population of cells with extensive self-renewal capacity (64). The self-renewing population, however, appears to be a minority population, as only $1 \%-2 \%$ of sphere cells form new spheres upon replating. Implantation of sphere cells in the renal capsule regeneration assay has shown that both primary and passaged sphere cells can regenerate prostate ductal structures containing both basal and luminal cells (64). These data suggest that murine PSCs are capable of extensive self-renewal and that this activity can be maintained using this prostate sphere assay.

\section{Identification and characterization of prostate epithelial stem cells}

Traditional approaches for isolation of tissue stem cells have utilized cell surface markers and cell-sorting technology. Using this approach, Collins and colleagues (70) demonstrated that prostate basal cells that rapidly adhere to type I collagen in vitro express high levels of $\alpha_{2} \beta_{1}$ integrin, possess greater colony-forming efficiency in vitro, and can produce prostate glandular structures 
when grafted onto the flanks of athymic mice. Subdivision of the $\alpha_{2} \beta_{1}{ }^{\text {hi }}$ basal cell compartment using CD133, a neural and hematopoietic stem cell marker, results in an 11-fold increase in colony formation (71). These $\mathrm{CD} 133^{+} \alpha_{2} \beta_{1}{ }^{\text {hi }}$ cells can also give rise to glandular tissue in vivo.

Independent studies by our lab and Burger et al. (72) have shown that Sca-1, a marker used to enrich for hematopoietic, mammary, and pulmonary stem cells, can be used to enrich for murine prostate cells with enhanced tubule-forming activity in vivo (61, $72-75)$. Sca- $1^{+}$cells possess several properties of stem cells, including multilineage differentiation capacity and replication quiescence (61). Sca-1 also appears to be present on the majority of primitive cells that remain following castration-induced apoptosis of mature prostate cells. We have also found that Sca- 1 is constitutively expressed by all epithelial cells of the fetal prostate, indicating that the marker is present on PSCs from very early stages in their development (62).

Wilson and colleagues demonstrated that Sca- $1^{+}$prostate cells express other stem cell markers such as BCL-2 and CD49f (integrin $\left.\alpha_{6}\right)(72)$. CD49f is a particularly prevalent stem cell marker that is expressed by hematopoietic, neural, embryonic, skin, and mammary epithelial stem cells (76-80). Magnetic bead sorting for $\mathrm{CD}_{49 \mathrm{f}^{+}}$cells enriches for cells with enhanced regenerative activity in vivo (30). We have shown that combining these markers to sort for cells with a $\mathrm{Lin}(\mathrm{CD} 45 / \mathrm{CD} 31 / \mathrm{Ter} 119)^{-} \mathrm{Sca}-1^{+} \mathrm{CD} 49 \mathrm{f}^{+}$(LSC) antigenic profile results in a 60 -fold enrichment for colony- and sphere-forming cells in vitro (64). These cells can self-renew to form spheres for many generations and can differentiate to produce ductal structures containing both basal and luminal cells in vivo. These studies estimate that PSCs can be isolated to a purity of up to 1 in 35 by using this antigenic profile.

Immunohistochemical staining of sorted LSC cells indicate that they possess a basal cell cytokeratin expression profile. LSC cells also localize to the basal cell layer within the proximal region of the gland, integrating both of the proposed models for PSC localization. The ability of LSC cells to produce $\mathrm{CK}^{+}$luminal cells in vivo also provides the first functional in vivo evidence for the traditional linear model for prostate epithelial cell differentiation, which proposes that basal cells give rise to luminal cells.

\section{Murine prostate cancer regeneration models}

Human cancers initiate from a wide range of genetic and epigenetic alterations. Studies in leukemia have shown that oncogenes induce tumorigenesis in some cell types more efficiently than in others, depending on their ability to confer self-renewal properties in the target cell (reviewed in ref. 9). Since the cellular source of prostate cancer may likewise be oncogene dependent, a long-term goal is to compare the tumorigenic activity of different prostate cell subpopulations following exposure to different oncogenic stimuli. Toward this end, we are using the dissociated prostate regeneration assay to investigate the tumorigenic effects of several prostate cancer-associated genetic alterations in PSCs as well as in more differentiated prostate cell populations.

The PTEN/AKT pathway. Loss of function of PTEN and activation of AKT are highly correlated with human prostate cancer (81, 82). Transgenic animals that express a constitutively active form of AKT under regulation of the prostate-specific probasin promoter develop PIN lesions (83). PTEN prostate-specific null animals also develop high-grade prostate adenocarcinomas and metastases $(54,84)$. We have found that lentivirus-mediated expression of constitutively active AKT in dissociated prostate cells results in the regeneration of prostate tubules containing PIN lesions that progress to frank carcinoma in some experiments $(61,85)$. PIN lesions also develop when prostate cells are infected with lentivirus carrying siRNA for targeted knockdown of PTEN (61).

The AR. Alterations in AR signaling causing receptor hypersensitivity, promiscuity, or androgen-independent receptor transactivation are also common features of prostate cancer (86). Interestingly, we find that overexpression of AR does not cause pathological growth but instead seems to reduce the tubule-forming activity of dissociated prostate cells in this assay (85). This is consistent with in vitro studies reporting that overexpression of AR reduces proliferation and promotes differentiation $(36,87)$. Lentivirus-mediated cell-autonomous overexpression of both AR and AKT, however, is sufficient to induce initiation and progression to frank carcinoma in this system. These regenerated tumors also progress to androgen independence, as continued growth is observed after several weeks of castration-induced androgen deprivation.

Investigation of tumorigenic activity of prostate cell subpopulations. In preliminary efforts to compare the tumorigenic activity of primitive and mature prostate cell populations, we isolated Sca- $1^{+}$and Sca- $1^{-}$cells and compared their response to overexpression of AKT1. We found that $\mathrm{Sca}-1^{+}$cells regenerate ductal structures that contain PIN, while Sca-1- cells give rise predominantly to normal ducts (61). AKT-regenerated tumors are marked by a 10 -fold expansion of the Sca- $1^{+}$population compared with normal regenerated prostate tissue. This expansion is also observed in prostate tumors that develop in PTEN-null animals (55). A future goal is to compare the oncogenic potential of LSCs and more differentiated cells following perturbation of the PTEN/ AKT pathway and AR signaling. Efforts to develop other models of prostate cancer are also underway using other prostate cancer-associated genetic alterations.

\section{Conclusions and implications}

Development of new therapeutics. Common anticancer treatments such as radiation and chemotherapy do not eradicate the majority of CSCs $(88,89)$. Several studies have shown that $\mathrm{Ph}^{+}$CSCs can still be identified in CML patients following imatinib mesylate treatment $(90,91)$. CSC resistance to these therapeutics may be mediated by several stem cell-related mechanisms, including replication quiescence, activation of antiapoptotic pathways, and multi-drug transporter expression. Androgen ablation therapies for invasive and metastatic prostate cancers may also spare prostate CSCs (47). Since mature prostate cells are dependent on androgen for viability, androgen ablation results in a dramatic tumor regression. However, progression to HRPC occurs quickly after treatment in nearly all patients. Many believe this is because, unlike normal PSCs, the prostate CSC population is not dependent on androgen for viability. Androgen ablation therapy may actually promote disease progression by stimulating normally quiescent CSCs to repopulate the tumor with primitive, blast-like androgen-independent cells. Research should therefore be aimed at developing therapeutics that can selectively target the CSC population rather than more differentiated prostate cancer cells. Clinical trials should likewise be designed to measure drug efficacy by examining their ability to eradicate CSCs rather than to measure bulk tumor regression.

Several strategies have been employed to eradicate CSCs. One involves targeting stem cell-signaling pathways to abrogate the self-renewal capacity of CSCs and induce differentiation. An inhib- 
itor of the Notch signaling pathway component, $\gamma$-secretase has recently been shown to have activity against mammary tumors that overexpress Notch1 $(92,93)$. Treatment with Hedgehog pathway inhibitors such as cyclopamine, anti-Hedgehog antibodies, and siRNAs against Gli have also been shown to abrogate the growth of medulloblastoma and prostate cancer in murine models (94-96). The challenge of these therapeutic approaches, however, is sparing normal tissue stem cells that utilize these pathways.

\section{Acknowledgments}

The authors thank Li Xin, Rita Lukacs, and Robert Signer for helpful suggestions about manuscript content. This work was supported by funds from the Prostate Cancer Foundation and the University of California, Los Angeles, Specialized Program of Research Excellence in prostate cancer (Jean deKernion, principal investigator). O.N. Witte is an Investigator at the Howard Hughes Medical Institute. D.A. Lawson is supported by NIH/Public Health Services Tumor Cell Biology Training grant T32 CA09056.

Address correspondence to: Owen N. Witte, Howard Hughes Medical Institute, University of California, Los Angeles, 675 Charles E. Young Dr. South, 5-748 MRL, Los Angeles, California 90095-1662, USA. Phone: (310) 206-0386; Fax: (310) 206-8822; E-mail: owenw@ microbio.ucla.edu.
1. Nowell, P.C., and Hungerford, D.A. 1960. Chromosome studies on normal and leukemic human leukocytes. J. Natl. Cancer Inst. 25:85-109.

2. Rowley, J.D. 1973. Letter: A new consistent chromosomal abnormality in chronic myelogenous leukaemia identified by quinacrine fluorescence and Giemsa staining. Nature. 243:290-293.

3. Wong, S., and Witte, O.N. 2004. The BCR-ABL story: bench to bedside and back. Annu. Rev. Immunol. 22:247-306.

4. Fialkow, P.J., Gartler, S.M., and Yoshida, A. 1967. Clonal origin of chronic myelocytic leukemia in man. Proc. Natl. Acad. Sci. U. S. A. 58:1468-1471.

5. Fialkow, P.J., Jacobson, R.J., and Papayannopoulou, T. 1977. Chronic myelocytic leukemia: clonal origin in a stem cell common to the granulocyte, erythrocyte, platelet and monocyte/macrophage. Am. J. Med. 63:125-130.

6. Takahashi, N., Miura, I., Saitoh, K., and Miura, A.B. 1998. Lineage involvement of stem cells bearing the philadelphia chromosome in chronic myeloid leukemia in the chronic phase as shown by a combination of fluorescence-activated cell sorting and fluorescence in situ hybridization. Blood. 92:4758-4763.

7. Kabarowski, J.H., and Witte, O.N. 2000. Consequences of BCR-ABL expression within the hematopoietic stem cell in chronic myeloid leukemia. Stem Cells. 18:399-408.

8. Reya, T., Morrison, S.J., Clarke, M.F., and Weissman, I.L. 2001. Stem cells, cancer, and cancer stem cells. Nature. 414:105-111.

9. Huntly, B.J., and Gilliland, D.G. 2005. Leukaemia stem cells and the evolution of cancer-stem-cell research. Nat. Rev. Cancer. 5:311-321.

10. Bonnet, D., and Dick, J.E. 1997. Human acute myeloid leukemia is organized as a hierarchy that originates from a primitive hematopoietic cell. Nat. Med. 3:730-737.

11. Huntly, B.J., et al. 2004. MOZ-TIF2, but not BCR$\mathrm{ABL}$, confers properties of leukemic stem cells to committed murine hematopoietic progenitors. Cancer Cell. 6:587-596.

12. Passegue, E., Wagner, E.F., and Weissman, I.L. 2004. JunB deficiency leads to a myeloproliferative disorder arising from hematopoietic stem cells. Cell. 119:431-443.

13. Krivtsov, A.V., et al. 2006. Transformation from committed progenitor to leukaemia stem cell initiated by MLL-AF9. Nature. 442:818-822.

14. Cozzio, A., et al. 2003. Similar MLL-associated leukemias arising from self-renewing stem cells and short-lived myeloid progenitors. Genes Dev. 17:3029-3035.

15. United States Cancer Statistics Working Group. 2006. United States cancer statistics: 2003 incidence and mortality. United States Department of Health and Human Services, Centers for Disease Control and Prevention, and National Cancer Institute. Atlanta, Georgia, USA. 451 pp. http://www.cdc.gov/cancer/npcr/uscs.

16. Tomlins, S.A., et al. 2005. Recurrent fusion of TMPRSS2 and ETS transcription factor genes in prostate cancer. Science. 310:644-648.

17. Bohlander, S.K. 2005. ETV6: a versatile player in leukemogenesis. Semin. Cancer Biol. 15:162-174.

18. Kovar, H. 2005. Context matters: the hen or egg problem in Ewing's sarcoma. Semin. Cancer Biol. 15:189-196.

19. Abate-Shen, C., and Shen, M.M. 2000. Molecular genetics of prostate cancer. Genes Dev. 14:2410-2434.

20. English, H.F., Santen, R.J., and Isaacs, J.T. 1987. Response of glandular versus basal rat ventral prostatic epithelial cells to androgen withdrawal and replacement. Prostate. 11:229-242.

21. Isaacs, J.T. 1985. Control of cell proliferation and cell death in the normal and neoplastic prostate: a stem cell model. In Benign prostatic byperplasia. C.H. Rodgers et al., editors. Department of Health and Human Services. Washington, DC, USA. NIH. Bethesda, Maryland, USA. 85-94.

22. Mills, A.A., Qi, Y., and Bradley, A. 2002. Conditional inactivation of $\mathrm{p} 63$ by Cre-mediated excision. Genesis. 32:138-141.

23. Mills, A.A., et al. 1999. p63 is a p53 homologue required for limb and epidermal morphogenesis. Nature. 398:708-713.

24. Yang, A., et al. 1999. p63 is essential for regenerative proliferation in limb, craniofacial and epithelial development. Nature. 398:714-718.

25. Signoretti, S., et al. 2000. p63 is a prostate basal cell marker and is required for prostate development. Am. J. Pathol. 157:1769-1775.

26. Signoretti, S., et al. 2005. p63 regulates commitment to the prostate cell lineage. Proc. Natl. Acad. Sci. U. S. A. 102:11355-11360.

27. Verhagen, A.P., et al. 1992. Colocalization of basal and luminal cell-type cytokeratins in human prostate cancer. Cancer Res. 52:6182-6187.

28. Tsujimura, A., et al. 2002. Proximal location of mouse prostate epithelial stem cells: a model of prostatic homeostasis. J. Cell Biol. 157:1257-1265.

29. Salm, S.N., et al. 2005. TGF-\{beta\} maintains dormancy of prostatic stem cells in the proximal region of ducts. J. Cell Biol. 170:81-90.

30. Goto, K., et al. 2006. Proximal prostatic stem cells are programmed to regenerate a proximal-distal ductal axis. Stem Cells. 24:1859-1868.

31. Litvinov, I.V., et al. 2006. Low-calcium serum-free defined medium selects for growth of normal prostatic epithelial stem cells. Cancer Res. 66:8598-8607.

32. Wang, Y., Hayward, S., Cao, M., Thayer, K., and Cunha, G. 2001. Cell differentiation lineage in the prostate. Differentiation. 68:270-279.

33. Xue, Y., Smedts, F., Debruyne, F.M., de la Rosette, J.J., and Schalken, J.A. 1998. Identification of intermediate cell types by keratin expression in the developing human prostate. Prostate. 34:292-301.

34. van Leenders, G., Dijkman, H., Hulsbergen-van de Kaa, C., Ruiter, D., and Schalken, J. 2000. Demonstration of intermediate cells during human prostate epithelial differentiation in situ and in vitro using triple-staining confocal scanning microscopy. Lab. Invest. 80:1251-1258.
35. Uzgare, A.R., Xu, Y., and Isaacs, J.T. 2004. In vitro culturing and characteristics of transit amplifying epithelial cells from human prostate tissue. J. Cell. Biochem. 91:196-205.

36. Garraway, L.A., et al. 2003. Intermediate basal cells of the prostate: in vitro and in vivo characterization. Prostate. 55:206-218.

37. Tokar, E.J., Ancrile, B.B., Cunha, G.R., and Webber, M.M. 2005. Stem/progenitor and intermediate cell types and the origin of human prostate cancer. Differentiation. 73:463-473.

38. Robinson, E.J., Neal, D.E., and Collins, A.T. 1998. Basal cells are progenitors of luminal cells in primary cultures of differentiating human prostatic epithelium. Prostate. 37:149-160.

39. Tran, C.P., Lin, C., Yamashiro, J., and Reiter, R.E. 2002. Prostate stem cell antigen is a marker of late intermediate prostate epithelial cells. Mol. Cancer Res. 1:113-121.

40. Liu, A.Y., et al. 1997. Cell-cell interaction in prostate gene regulation and cytodifferentiation. Proc. Natl. Acad. Sci. U. S. A. 94:10705-10710.

41. Visvader, J.E., and Lindeman, G.J. 2006. Mammary stem cells and mammopoiesis. Cancer Res. 66:9798-9801.

42. Kurita, T., Medina, R.T., Mills, A.A., and Cunha, G.R. 2004. Role of p63 and basal cells in the prostate. Development. 131:4955-4964.

43. Asselin-Labat, M.L., et al. 2007. Gata-3 is an essential regulator of mammary-gland morphogenesis and luminal-cell differentiation. Nat. Cell Biol. 9:201-209.

44. Abrahamsson, P.A. 1999. Neuroendocrine differentiation in prostatic carcinoma. Prostate. 39:135-148.

45. Okada, H., et al. 1992. Keratin profiles in normal/ hyperplastic prostates and prostate carcinoma. Virchows Arch. A Pathol. Anat. Histopathol. 421:157-161.

46. Reiter, R.E., et al. 1998. Prostate stem cell antigen: a cell surface marker overexpressed in prostate cancer. Proc. Natl. Acad. Sci. U. S. A. 95:1735-1740.

47. Litvinov, I.V., De Marzo, A.M., and Isaacs, J.T. 2003. Is the Achilles' heel for prostate cancer therapy a gain of function in androgen receptor signaling? J. Clin. Endocrinol. Metab. 88:2972-2982.

48. Collins, A.T., Berry, P.A., Hyde, C., Stower, M.J., and Maitland, N.J. 2005. Prospective identification of tumorigenic prostate cancer stem cells. Cancer Res. 65:10946-10951.

49. Richardson, G.D., et al. 2004. CD133, a novel marker for human prostatic epithelial stem cells. J. Cell Sci. 117:3539-3545.

50. Patrawala, L., et al. 2006. Highly purified CD44+ prostate cancer cells from xenograft human tumors are enriched in tumorigenic and metastatic progenitor cells. Oncogene. 25:1696-1708.

51. Gu, G., Yuan, J., Wills, M., and Kasper, S. 2007. Prostate cancer cells with stem cell characteristics reconstitute the original human tumor in vivo. Cancer Res. 67:4807-4815.

52. Miki, J., et al. 2007. Identification of putative stem cell markers, CD133 and CXCR4, in hTERT- 
immortalized primary nonmalignant and malignant tumor-derived human prostate epithelial cell lines and in prostate cancer specimens. Cancer Res. 67:3153-3161.

53. Cerveira, N., et al. 2006. TMPRSS2-ERG gene fusion causing ERG overexpression precedes chromosome copy number changes in prostate carcinomas and paired HGPIN lesions. Neoplasia. 8:826-832.

54. Wang, S., et al. 2003. Prostate-specific deletion of the murine Pten tumor suppressor gene leads to metastatic prostate cancer. Cancer Cell. 4:209-221.

55. Wang, S., et al. 2006. Pten deletion leads to the expansion of a prostatic stem/progenitor cell subpopulation and tumor initiation. Proc. Natl. Acad. Sci.U.S. A. 103:1480-1485.

56. Zhou, Z., Flesken-Nikitin, A., and Nikitin, A.Y. 2007. Prostate cancer associated with $\mathrm{p} 53$ and $\mathrm{Rb}$ deficiency arises from the stem/progenitor cellenriched proximal region of prostatic ducts. Cancer Res. 67:5683-5690.

57. Cunha, G.R., and Lung, B. 1978. The possible influence of temporal factors in androgenic responsiveness of urogenital tissue recombinants from wildtype and androgen-insensitive (Tfm) mice. J. Exp. Zool. 205:181-193.

58. Xin, L., Ide, H., Kim, Y., Dubey, P., and Witte, O.N. 2003. In vivo regeneration of murine prostate from dissociated cell populations of postnatal epithelia and urogenital sinus mesenchyme. Proc. Natl. Acad. Sci.U. S. A. 100(Suppl. 1):11896-11903.

59. Collins, A.T., Habib, F.K., Maitland, N.J., and Neal, D.E. 2001. Identification and isolation of human prostate epithelial stem cells based on alpha(2)beta(1)-integrin expression. J. Cell Sci. 114:3865-3872.

60. Azuma, M., et al. 2005. A quantitative matrigel assay for assessing repopulating capacity of prostate stem cells. Biochem. Biophys. Res. Commun. 338:1164-1170.

61. Xin, L., Lawson, D.A., and Witte, O.N. 2005. The Sca-1 cell surface marker enriches for a prostateregenerating cell subpopulation that can initiate prostate tumorigenesis. Proc. Natl. Acad. Sci. U. S. A. 102:6942-6947.

62. Lawson, D.A., et al. 2005. Prostate stem cells and prostate cancer. Cold Spring Harb. Symp. Quant. Biol. 70:187-196.

63. Hudson, D.L., O'Hare, M., Watt, F.M., and Masters, J.R. 2000. Proliferative heterogeneity in the human prostate: evidence for epithelial stem cells. $L a b$. Invest. 80:1243-1250.

64. Lawson, D.A., Xin, L., Lukacs, R.U., Cheng, D., and Witte, O.N. 2007. Isolation and functional characterization of murine prostate stem cells. Proc. Natl. Acad. Sci. U. S. A. 104:181-186.

65. Reynolds, B.A., and Weiss, S. 1996. Clonal and population analyses demonstrate that an EGFresponsive mammalian embryonic CNS precursor is a stem cell. Dev. Biol. 175:1-13.

66. Dontu, G., et al. 2003. In vitro propagation and transcriptional profiling of human mammary stem/progenitor cells. Genes Dev. 17:1253-1270.

67. Webber, M.M., Bello, D., Kleinman, H.K., and Hoffman, M.P. 1997. Acinar differentiation by non-malignant immortalized human prostatic epithelial cells and its loss by malignant cells. Carcinogenesis. 18:1225-1231.

68. Bello-DeOcampo, D., Kleinman, H.K., Deocampo, N.D., and Webber, M.M. 2001. Laminin-1 and alpha6beta 1 integrin regulate acinar morphogenesis of normal and malignant human prostate epithelial cells. Prostate. 46:142-153.

69. Lang, S.H., Sharrard, R.M., Stark, M., Villette, J.M., and Maitland, N.J. 2001. Prostate epithelial cell lines form spheroids with evidence of glandular differentiation in three-dimensional Matrigel cultures. Br. J. Cancer. 85:590-599.

70. Collins, A.T., Habib, F.K., Maitland, N.J., and Neal, D.E. 2001. Identification and isolation of human prostate epithelial stem cells based on alpha(2)beta(1)-integrin expression. J. Cell Sci. 114:3865-3872.

71. Richardson, G.D., et al. 2004. CD133, a novel marker for human prostatic epithelial stem cells. J. Cell Sci. 117:3539-3545

72. Burger, P.E., et al. 2005. Sca-1 expression identifies stem cells in the proximal region of prostatic ducts with high capacity to reconstitute prostatic tissue. Proc. Natl. Acad. Sci. U. S. A. 102:7180-7185.

73. Welm, B.E., et al. 2002. Sca-1(pos) cells in the mouse mammary gland represent an enriched progenitor cell population. Dev. Biol. 245:42-56.

74. Spangrude, G.J., Heimfeld, S., and Weissman, I.L. 1988. Purification and characterization of mouse hematopoietic stem cells. Science. 241:58-62.

75. Kim, C.F., et al. 2005. Identification of bronchioalveolar stem cells in normal lung and lung cancer. Cell. 121:823-835.

76. Stingl, J., et al. 2006. Purification and unique properties of mammary epithelial stem cells. Nature. 439:993-997.

77. Tani, H., Morris, R.J., and Kaur, P. 2000. Enrichment for murine keratinocyte stem cells based on cell surface phenotype. Proc. Natl. Acad. Sci. U. S. A. 97:10960-10965.

78. Fortunel, N.O., et al. 2003. Comment on " 'Stemness': transcriptional profiling of embryonic and adult stem cells" and "a stem cell molecular signature". Science. 302:393; author reply 393.

79. Ramalho-Santos, M., Yoon, S., Matsuzaki, Y., Mulligan, R.C., and Melton, D.A. 2002. "Stemness": transcriptional profiling of embryonic and adult stem cells. Science. 298:597-600.

80. Ivanova, N.B., et al. 2002. A stem cell molecular signature. Science. 298:601-604.
81. Deocampo, N.D., Huang, H., and Tindall, D.J. 2003. The role of PTEN in the progression and survival of prostate cancer. Minerva Endocrinol. 28:145-153.

82. Sun, M., et al. 2001. AKT1/PKBalpha kinase is frequently elevated in human cancers and its constitutive activation is required for oncogenic transformation in NIH3T cells. Am. J. Pathol. 159:431-437.

83. Majumder, P.K., et al. 2003. Prostate intraepithelial neoplasia induced by prostate restricted Akt activation: the MPAKT model. Proc. Natl. Acad. Sci. U. S. A. 100:7841-7846

84. Ma, X., et al. 2005. Targeted biallelic inactivation of Pten in the mouse prostate leads to prostate cancer accompanied by increased epithelial cell proliferation but not by reduced apoptosis. Cancer Res. 65:5730-5739.

85. Xin, L., et al. 2006. Progression of prostate cancer by synergy of AKT with genotropic and nongenotropic actions of the androgen receptor. Proc. Natl. Acad. Sci. U. S. A. 103:7789-7794.

86. Feldman, B.J., and Feldman, D. 2001. The development of androgen-independent prostate cancer. Nat. Rev. Cancer. 1:34-45.

87. Berger, R., et al. 2004. Androgen-induced differentiation and tumorigenicity of human prostate epithelial cells. Cancer Res. 64:8867-8875.

88. Guzman, M.L., et al. 2002. Preferential induction of apoptosis for primary human leukemic stem cells. Proc. Natl. Acad. Sci. U. S. A. 99:16220-16225.

89. Jones, R.J., Matsui, W.H., and Smith, B.D. 2004. Cancer stem cells: are we missing the target? J. Natl. Cancer Inst. 96:583-585.

90. Bhatia, R., et al. 2003. Persistence of malignant hematopoietic progenitors in chronic myelogenous leukemia patients in complete cytogenetic remission following imatinib mesylate treatment. Blood. 101:4701-4707.

91. Graham, S.M., et al. 2002. Primitive, quiescent, Philadelphia-positive stem cells from patients with chronic myeloid leukemia are insensitive to STI571 in vitro. Blood. 99:319-325.

92. Weijzen, S., et al. 2002. Activation of Notch-1 signaling maintains the neoplastic phenotype in human Ras-transformed cells. Nat. Med. 8:979-986.

93. Pece, S., et al. 2004. Loss of negative regulation by Numb over Notch is relevant to human breast carcinogenesis. J. Cell Biol. 167:215-221.

94. Romer, J.T., et al. 2004. Suppression of the Shh pathway using a small molecule inhibitor eliminates medulloblastoma in Ptc1(+/-)p53(-/-) mice. Cancer Cell. 6:229-240.

95. Karhadkar, S.S., et al. 2004. Hedgehog signalling in prostate regeneration, neoplasia and metastasis. Nature. 431:707-712.

96. Stecca, B., Mas, C., and Ruiz i Altaba, A. 2005. Interference with HH-GLI signaling inhibits prostate cancer. Trends Mol. Med. 11:199-203. 